Proceedings of the International School and Conference on Optics and Optical Materials, ISCOM07, Belgrade, Serbia, September 3-7, 2007

\title{
Surface Optical Waves in Semi-Infinite One-Dimensional Photonic Crystals Containing Alternating Layers of Positive and Negative Media with a Cap Layer
}

\author{
J. BARvestani ${ }^{a, b, *}$, M. KAlafi ${ }^{a, b, c}$ AND A. Soltani-VAla ${ }^{a, b}$ \\ ${ }^{a}$ Research Institute for Applied Physics and Astronomy \\ University of Tabriz, Tabriz 51665-163, Iran \\ ${ }^{b}$ Physics Faculty, University of Tabriz, Iran \\ ${ }^{c}$ Excellence Center for Photonics, University of Tabriz, Iran
}

\begin{abstract}
An analytical direct matching procedure within the Kronig-Penney model was applied to analyze the dispersion behavior of the localized surface states supported at the surface of a semi-infinite one-dimensional photonic crystals truncated with air or a cap layer. The photonic crystals contain alternating layers of positive and negative media. The present study demonstrates that by choosing some proper value for the photonic crystal parameters, zero and negative dispersion of surface modes emerge in a large range of parallel wave vector. New forward and backward surface waves are introduced. Due to the different nature of the band structure the surface modes are more localized compared to those appearing in the conventional photonic crystals.
\end{abstract}

PACS numbers: 42.70.Qs, 73.20.At, 63.20.Pw, 68.65.Cd

\section{Introduction}

Apart from their intrinsic interest, surface electromagnetic waves have recently been proposed as a way to efficiently inject light into a photonic crystal (PC) waveguide, or to extract a focused beam from a channel [1-4]. It is well known that when an ideal infinite PC is truncated, localized surface waves can appear on the truncation surface of semi-infinite PC. These modes are electromagnetic fields that decay exponentially along the normal direction away from the surface into both the bulk crystals and the homogeneous background [5-8].

*corresponding author; e-mail: barvestani@tabrizu.ac.ir 
Surface modes have become a familiar physical concept in the optics and physics community thanks to the long history of investigation on surface plasmons, a kind of localized surface wave that is typically excited in metal films. Surface plasmons have already found use in a wide variety of areas such as nonlinear optics, optical modulators, and sensors $[9,10]$. In the case of the conventional materials the surface modes shift into higher frequencies as the incident light changes from normal to oblique incidence. For this reason, the phenomenon of photonic band gap has been used only under a narrow range of frequencies of light incident at a particular angle or in a particular angular range.

In this paper, we have theoretically investigated localized surface states supported at the surface of a semi-infinite 1D PC containing alternating layers of left handed and right handed materials (referred to as $\mathrm{L}-\mathrm{R}$ PC throughout this paper). To the best of our knowledge, there is no report regards to the surface modes in L-R PC. Left handed materials in which the dielectric permittivity $\varepsilon$ and magnetic permeability $\mu$ are simultaneously negative, have received a great deal of attention during the last few years [11]. In our calculations, the dielectric permittivity and magnetic permeability are, in general, assumed to take constant values. Although these parameters in left handed materials are in general frequency dependent, our results can be used to design specific metamaterials that would lead to a typical behavior around a given frequency. We have demonstrated that choosing an appropriate truncation and cap layer parameters in L-R PC leads to a type of surface modes which possesses such properties as independence of the incident angles. This kind of dispersion has been reported in the conventional 1D $\mathrm{PC}$ containing a defect layer with a negative refraction index [12]. The zero and negative dispersion phenomena may be useful in the design of large incident angle filters and narrow frequency and sharp angular filters, respectively [12].

\section{The basic equations}

In order to obtain the surface states in 1D PCs, the direct matching procedure within the Kronig-Penney model have been used [13]. Let us suppose each unit cell is composed of two layers, which are stacked along the $z$-axis direction where $d_{i}$ and $\varepsilon_{i}$ are the thickness and the dielectric constant of the $i$-th layer, respectively. Due to translational invariance in the $X O Y$ plane, the parallel wave vector $k_{\|}$is a conservative quantity in all domains of the crystal. We assume that the $z$-axis is along the normal to the layers and $k_{\|}$is along the $x$-axis. Now we consider configuration which is displayed in Fig. 1.

The dispersion relation of the $\mathrm{PC}$ has the well-known form

$$
\begin{aligned}
& \cos (k d)=\cosh \left(k_{1 z} d_{1}\right) \cos \left(k_{2 z} d_{2}\right)+\frac{1}{2}\left(\frac{F_{1}}{F_{2}}+\frac{F_{2}}{F_{1}}\right) \sinh \left(k_{1 z} d_{1}\right) \sinh \left(k_{2 z} d_{2}\right) \\
& \quad=B(\omega),
\end{aligned}
$$

where the perpendicular wave vector component in each medium is given by $k_{i z}=\left[k_{\|}^{2}-\varepsilon_{i} \mu_{i}(\omega / c)^{2}\right]^{1 / 2}, F_{i}=k_{i z} / \mu_{i}(i=1,2), k_{\|}^{2}=k_{x}^{2}+k_{y}^{2}, \omega$ is the an- 

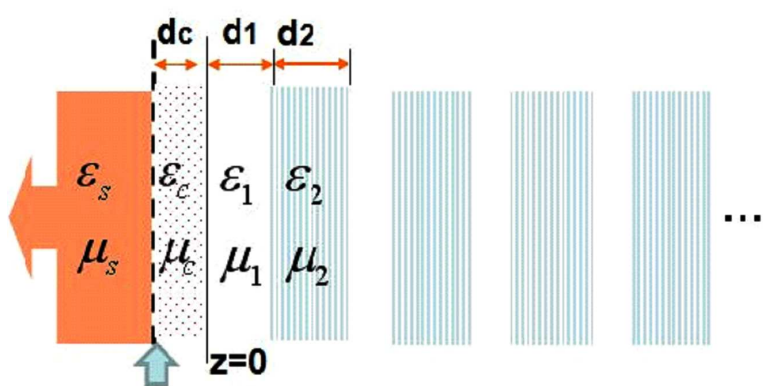

Fig. 1. Schematic representation of configuration. The blue arrow shows the position of surface plane.

gular frequency, $d=d_{1}+d_{2}$ is the lattice constant, and $c$ is the light speed in vacuum [11]. It is well known that when any periodic system is limited, $k$ should be complex

$$
k=\mathrm{i} \eta+\frac{n \pi}{d}, \eta>0, n=0, \pm 1, \pm 2, \ldots
$$

It is straightforward to write the electromagnetic field of TE polarization under the form

$$
\begin{cases}E_{y}^{\mathrm{s}}=A_{\mathrm{s}} \mathrm{e}^{\mathrm{i} k_{\mathrm{s}} z} \mathrm{e}^{\mathrm{i}\left(k_{\|} x-\omega t\right)}, & -\infty<z \leq-d_{\mathrm{c}}, \\ E_{y}^{\mathrm{c}}=\left(A_{\mathrm{c}} \sinh k_{\mathrm{c}} z+B_{\mathrm{c}} \cosh k_{\mathrm{c}} z\right) \mathrm{e}^{\mathrm{i}\left(k_{\|} x-\omega t\right)}, & -d_{\mathrm{c}} \leq z \leq 0, \\ E_{y}^{\mathrm{PC}}=C_{1}\left(\sinh k_{1} z+\gamma \cosh k_{1} z\right) \mathrm{e}^{\mathrm{i}\left(k_{\|} x-\omega t\right)}, & 0 \leq z \leq d_{1},\end{cases}
$$

where $\gamma=\left[\sinh \left(k_{1 z} d_{1}\right)+\frac{F 1}{F 2} \mathrm{e}^{\mathrm{i} k d} \sinh \left(k_{2 z} d_{2}\right)\right] /\left[\mathrm{e}^{\mathrm{i} k d} \cosh \left(k_{2 z} d_{2}\right)-\cosh \left(k_{1 z} d_{1}\right)\right]$, $k_{\mathrm{s}}=\left[k_{\|}^{2}-\varepsilon_{\mathrm{s}} \mu_{\mathrm{s}}(\omega / c)^{2}\right]^{1 / 2}, k_{\mathrm{c}}=\left[k_{\|}^{2}-\varepsilon_{\mathrm{c}} \mu_{\mathrm{c}}(\omega / c)^{2}\right]^{1 / 2}$. Here, $\mathrm{s}$ and $\mathrm{c}$ indices represent the homogeneous semi-infinite media and cap layer, respectively. Using the boundary conditions at the $z=0$ and $z=-d_{\mathrm{c}}$, one easily obtains the equation giving the surface modes dispersion relation

$$
\frac{F_{1}}{\gamma} \cosh k_{\mathrm{c}} d_{\mathrm{c}}-F_{\mathrm{s}} \cosh k_{\mathrm{c}} d_{\mathrm{c}}-F_{\mathrm{c}} \sinh k_{\mathrm{c}} d_{\mathrm{c}}+\frac{F_{1} F_{\mathrm{s}}}{F_{\mathrm{c}} \gamma} k_{\mathrm{c}} d_{\mathrm{c}}=0,
$$

where $F_{\mathrm{s}}=k_{\mathrm{s}} / \mu_{\mathrm{s}}$ and $F_{\mathrm{c}}=k_{\mathrm{c}} / \mu_{\mathrm{c}}$. In the absent of the cap layer $\left(d_{\mathrm{c}}=0\right)$, this equation reduces to $\frac{F_{1}}{\gamma}-F_{\mathrm{s}}=0$, see Ref. [14].

The surface modes decay exponentially along the normal direction away from the surface into both the bulk crystals and the homogeneous background. By eliminating $\eta$ between (1) and (4) and using of Eq. (2), we get the following relation:

$$
S(\omega)=B(\omega) \mp\left[B^{2}(\omega)-1\right]^{1 / 2}, \quad S(\omega)=(-1) \mathrm{e}^{-\eta d},
$$

where - and + correspond to even and odd $n$, respectively [15]. By applying this procedure, some spurious solutions are introduced. This can be identified and should be rejected by applying existence condition, i. e., $\mathrm{e}^{-\eta d}<1[16]$. 


\section{Results and discussion}

The surface waves are forward or backward when the total energy flux is positive or negative, respectively [17]. The total energy flux in the RH and LH media is an integral of the Poynting vector over the corresponding spatial regions. The energy flow is described by the Poynting vector, which defines the energy density flux averaged over the period $T=2 \pi / \omega$, and can be written in the form

$$
-\frac{\partial U}{\partial t}=\int \boldsymbol{\nabla} \cdot \boldsymbol{S} \mathrm{d} V, \quad \boldsymbol{S}=\frac{c}{8 \pi} \operatorname{Re}\left[\boldsymbol{E} \times \boldsymbol{H}^{*}\right],
$$

where $\boldsymbol{E}, \boldsymbol{H}$ are the electric and magnetic field of the surface wave, respectively, $U$ is the total energy and the asterisk stands for the complex conjugation.
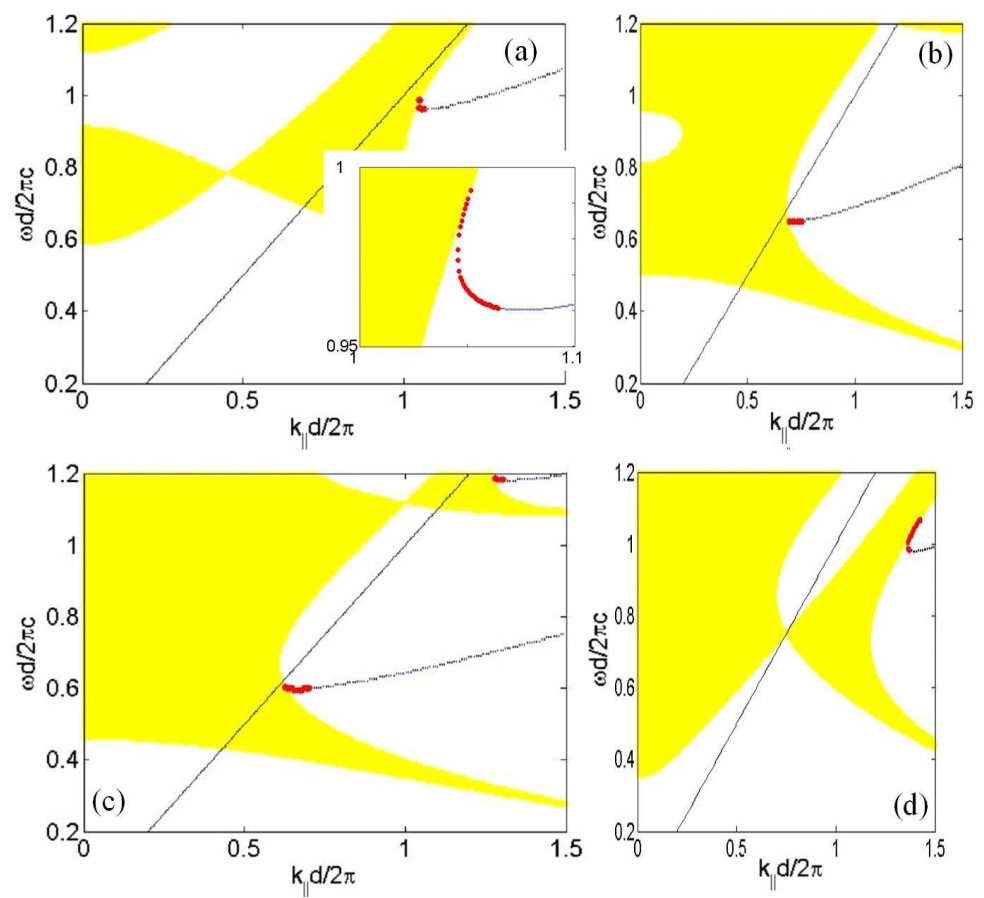

Fig. 2. Calculated bulk and surface modes for TE polarization in a semi-infinite 1D L-R PC. The physical parameters used are in (a) $\varepsilon_{1}=4$, (b) $\varepsilon_{1}=7$, (c) $\varepsilon_{1}=8$, and in $(\mathrm{d})$ are $d_{1}=d / 3, \varepsilon_{1}=5, \mu_{1}=1 ; d_{2}=2 d / 3, \varepsilon_{2}=-1.25$, and $\mu_{2}=-2$. In all cases $d_{\mathrm{c}}=0$. The gray and white regions are pass and forbidden bands, respectively. The bold and thin curves in the gaps show the backward and forward modes, respectively. The straight bold line shows the light line of homogeneous medium with $\varepsilon_{\mathrm{s}}=\mu_{\mathrm{s}}=1$. The inset in part (a) shows the magnified region of surface modes.

Firstly, we studied the behavior of the surface states of photonic crystal without a cap layer $\left(d_{\mathrm{c}}=0\right)$ and the first layer of photonic crystal is right handed material. We choose the following parameters for the structure: $d_{1}=d / 3, \mu_{1}=1$; 
$d_{2}=2 d / 3, \varepsilon_{2}=-1.25$ and $\mu_{2}=-1$. Band structures and dispersion of surface modes are displayed in Fig. 2 for (a) $\varepsilon_{1}=4$, (b) $\varepsilon_{1}=7$, (c) $\varepsilon_{1}=8$, and physical parameters of Fig. $2 \mathrm{~d}$ are $d_{1}=d / 3, \varepsilon_{1}=5, \mu_{1}=1 ; d_{2}=2 d / 3, \varepsilon_{2}=-1.25$, and $\mu_{2}=-2$. We can see from these figures that the dispersion of surface states is positive, except near the band edges, that is interpreted later, which is similar to the surface modes in conventional PC, but, in contrary to the conventional PC, these states are far away from band edges and consequently the surface modes become more localized. Near the band edges and in narrow range of $k_{\|}$there is a new unusual effect, i.e. zero and negative slope of dispersion curve. It must be noted that the slope of dispersion curve for the conventional PC are always positive, while it may be negative for $\mathrm{L}-\mathrm{R} \mathrm{PC}$ due to the presence of left-handed material (LHM). There are modes with negative and positive group velocities which are termed as backward and forward, respectively. In the forward wave, the direction of the Poynting vector of the forward wave coincides with the propagation direction, while in the backward wave the Poynting vector is backward with respect to the wave vector. Thus, there is a flexible control of the dispersion properties of surface modes and band structures by varying the physical and the structural parameters of the $\mathrm{L}-\mathrm{R}$ PC.

In the next structure we choose the following parameters: $d_{1}=2 d / 3$, $\varepsilon_{1}=-1.25, \mu_{1}=-1 ; d_{2}=d / 3, \varepsilon_{2}=5, \mu_{2}=1 ; d_{\mathrm{c}}=0.3 d, \varepsilon_{\mathrm{c}}=3, \mu_{\mathrm{c}}=1$, indeed we interchanged the positions of two layers of PC, so that the first layer is a LHM. We can see from Fig. 3 that the backward modes also exist in this case. It must be noted that in the absence of cap layer and for these choices of physical parameters, these modes are absent and we can produce them by suitable choice of physical parameters of cap layer.

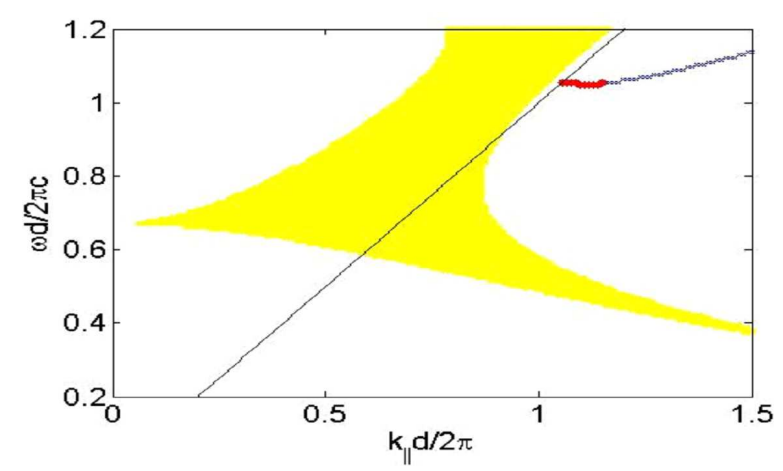

Fig. 3. The same as in Fig. 2 and the parameters are $d_{1}=2 d / 3, \varepsilon_{1}=-1.25, \mu_{1}=-1$; $d_{2}=d / 3, \varepsilon_{2}=5, \mu_{2}=1 ; d_{\mathrm{c}}=0.3 d, \varepsilon_{\mathrm{c}}=3, \mu_{\mathrm{c}}=1$. By these choices the average index of refraction in the $\mathrm{PC},\langle n\rangle=\left(n_{1} d_{1}+n_{2} d_{2}\right) / d$, is zero and we have discrete bands in $k_{\|}=0$.

In many applications involving the surface modes, we almost need the states strengthly localized within the band gap. It is well known that the strengthest 
localization corresponds to a surface mode located at the midgap. Thus, by choosing an appropriate value for physical parameters of L-R PC and cap layer, it will be possible to obtain a surface mode with a desired localization strength.

\section{Conclusion}

The direct matching procedure was applied to obtain the localized surface optical modes in a semi-infinite 1D PC containing alternating layers of positive and negative media. We demonstrated that stacking alternating layers of positive and negative with a cap layer leads to a type of surface modes which possess zero and negative dispersion. Moreover, we have shown that in the presence of the left-handed metamaterial in photonic crystal, the surface modes can be either forward or backward while for conventional structures the surface modes are always forward.

\section{References}

[1] E. Moreno, L. Martin Moreno, F.J. Garcia-Vidal, Phys. Rev. B 69, 121402R (2004).

[2] E. Moreno, L. Martin Moreno, F.J. Garcia-Vidal, Phot. Nanostr. 2, 97 (2004).

[3] P. Kramper, M. Agio, C.M. Soukoulis, A. Bimer, F. Muller, Wehrspohn, U. Gosele, V. Sandoghdar, Phys. Rev. Lett. 92, 113903 (2003).

[4] A.I. Rahachou, Zozoulenko, arxiv:physics/0510273.

[5] R.D. Meade, K.D. Brommer, A.M. Rappe, J.D. Joannopoulos, Phys. Rev. B 44, 10961 (1991).

[6] W.M. Robertson, M.S. May, Appl. Phys. Lett. 74, 1800 (1999).

[7] F. Ramos-Mendieta, P. Halvei, Phys. Rev. B 59, 15112 (1999).

[8] M. Qiu, S. He, Phys. Lett. A 282, 85 (2001).

[9] L.L. Lin, Z.Y. Li, Phys. Rev. B 63, 033310 (2001).

[10] O. Solgaard, F. Ho, J.I. Thackara, D.M. Bloom, Appl. Phys. Lett. 61, 2500 (1992).

[11] D. Bria, B. Djafari-Rouhani, A. Akjouj, L. Dobrzynski, J.P. Vigneron, E.H. El Boudouti, A. Nougaui, Phys. Rev. E 69, 066613 (2004).

[12] G. Liang, P. Han, H. Wang, Opt. Lett. 29, 192 (2004).

[13] M. Steslicka, R. Kucharczyk, A. Akjouj, B. Djafari-Rouhani, L. Dobrzynski, S.G. Davison, Surf. Sc. Reports 47, 93 (2002).

[14] M. Kalafi, A. Soltani-Vala, J. Barvestani, Opt. Commun. 272, 403 (2007).

[15] M. Steslicka, R. Kucharczyk, M.L. Glasser, Phys. Rev. B 42, 1458 (1990).

[16] M. Steslicka, R. Kucharczyk, L. Dobrzynski, B. Djafari-Rouhani, E.H. El Boudouti, W. Jaskolski, Prog. Surf. Sci. 46, 219 (1994).

[17] I. Shadrivov, A. Soukhorukov, Y. Kivshar, Phys. Rev. E 69, 016617 (2004). 